\title{
Sample concentration in a microfluidic paper-based analytical device using ion
}

concentration polarization

Dinh-Tuan Phan, ${ }^{\mathrm{a}}$ Seyed Ali Mousavi Shaegh, ${ }^{\mathrm{b}}$ Yang Chun, ${ }^{{ }_{\mathrm{a}}}$ and Nam-Trung Nguyen ${ }^{{ }^{*} \mathrm{c}}$

We report a low-cost approach for sample concentration in a microfluidic paperbased analytical device using ion concentration polarization. This device platform can attain liquid sample filling by capillary suction through the microporous paper and ion selective transport through a nanoporous Nafion membrane under a DC electric field. The device is demonstrated for a fast depletion of fluorescent dye samples and an effective microfluidic concentrator for fluorescent dye with about 40-fold concentration enhancement achieved in 200 seconds. The device fabrication is simply based on paper cutting and lamination without need of lithography and printing of hydrophobic material such as wax. The lamination approach presented in this paper has the potential to be transferred to a large-scale production of paper-based analytical devices.

\section{Introduction}

Ion concentration polarization (ICP) is an ionic transport phenomenon that occurs near an ion-selective membrane and includes the ion-depletion and ion-enrichment processes. ${ }^{1}$ Recently, ICP has attracted a great attention from the microfluidics research community due to its potential applications in sample concentration ${ }^{2-5}$, desalination $^{6,7}$, and mixing. ${ }^{8,9}$ Concentration of low-abundance analytes is a critical need for microfluidic point-of-care portable analytical devices, because subsequent analysis or detection is only possible at analyte concentrations above the limit of detection of the analytical device. Several research groups have explored various concentration methods including field amplification stacking, ${ }^{10}$ isoelectric focusing, ${ }^{11}$ membrane filtration, ${ }^{12}$ affinity-based extraction ${ }^{13}$, temperature gradient focusing with a combined AC and DC field ${ }^{14}$ and electrokinetic trapping based on ion concentration polarization (ICP). ${ }^{15}$

ICP-based electrokinetic trapping method has numerous unique features. Specifically, concentration of molecules with ICP is independent of the hydrophobicity of binding characteristics of the molecules of interest. ${ }^{2}$ In addition, an electric field created by simple electrodes can easily manipulate the concentrating process. ${ }^{2}$ This allows ICP-based concentrators to be integrated in a wide range of microfluidic platforms for detection of target molecules initially at a low concentration.

Conventionally, ICP is observed at the interface of a permselective nanochannel bridging two adjacent microchannels. ${ }^{16}$ Once an ICP is established; a depletion zone is formed at one side of the perm-selective nanochannel. In the ion depletion zone, the concentration of both negatively and positively charged species drops sharply. In contrast, on the other side of the nanochannel, an ion enrichment zone is established with a high concentration of both negatively and positively charged species. ${ }^{2}$ 
Nanoporous ion-selective materials with non-engineered pores have been used to make ICP-based microfluidic separators and concentrators. ${ }^{2}$ Nafion is for instance an ion-selective material with negatively-charged sulfonic groups. Kim et al. used a Nafion membrane to generate ICP for continuous seawater desalination. Nafion-based nanojunction was integrated at the bifurcated point of a microchannel where all charged species, particles and microorganisms were repelled into the brine channel and thus pure water was guided to the desalted channel. ${ }^{6}$ Kwak et al. adopted a similar approach for continuous-flow concentration of biomolecules, bacteria, and red blood cells. ${ }^{5}$ In both designs, the nano junction had to be in contact with an additional buffer channel, which added complexity to the chip design. Ko et al. reported an improved device for protein preconcentration. ${ }^{4}$ In their device, the Nafion membrane was placed at the bottom of a straight microchannel. With this design, the buffer channel was eliminated, resulting in a simplified fabrication process. Both anode and cathode were located in the same microchannel with one inlet and one outlet. The preconcentrator could enhance the detection sensitivity of the immunoassay for a sample of C-reactive protein by more than 500 -fold compared to an immunoassay without preconcentrator.

Recently, paper-based analytical microfluidics for point-of-care applications has also attracted a great attention. ${ }^{17-19}$ The low cost of material, fabrication and operation of paper-based devices makes the applications extremely suitable for resource-limited regions. ${ }^{20}$ Passive capillary wetting (suction) through the porous structure of paper makes fluid handling an easy task. In addition, the porous structure of paper provides a suitable matrix for effective immobilization of reagents through physical adsorption. ${ }^{21}$ Having such unique properties, various analytical assays have been implemented in the paper-based microfluidic platforms; for instance colorimetric detection, ${ }^{22}$ direct electrochemical detection ${ }^{19}$ and electrogenerated chemiluminescence detection ${ }^{23}$ are a few to name. The use of a concentrator is inevitable to further improve the detection limit of these analytical methods. Preconcentration process needs flow manipulation, which is challenging since valves should operate without instrumented actuators and controllers. ${ }^{24}$ Gong et al. recently integrated a Nafion membrane into a paper strip to make an ICP-based concentrator. ${ }^{25}$ The device could concentrate a fluorescent tracer up to 40-fold in a fully wet paper in a stamp-like device with the nanoporous membrane separated from the paper-based assay.

This paper presents the development of a paper-based device platform using simple integration of microporous and nanoporous materials for liquid handling and sample concentration. The device platform functions for liquid sample filling by capillary suction through the microporous paper and ion selective transport through the nanoporous polymer matrix (Nafion membrane) under DC electric field. The functionality of this platform is demonstrated by ICP-based microfluidic sample depletion and concentration. Our work is distinct from other published works due to the simpler geometry with maintaining functionality, fast fabrication process thus leading to low cost, ease of applicability for practical use.

\section{Experimental section}

\section{Device concept and fabrication}

Figure 1a shows the concept of the concentration device. A paper strip forms two reservoirs and a main channel for introducing the sample. A thin Nafion membrane is 
put at the bottom of the paper strip. While the microporous paper represents the microfluidic channels for sample transport, the nanoporous Nafion membrane represents the nano-channels that are ion selective. Two electrodes are placed in the reservoirs at the two ends of the paper strip for producing two different DC voltages. A platinum wire acting as the ground electrode is placed under the Nafion membrane to activate the transport process across the nonporous matrix. This simple device configuration will demonstrate that sample depletion and concentration can be achieved on a single paper strip.

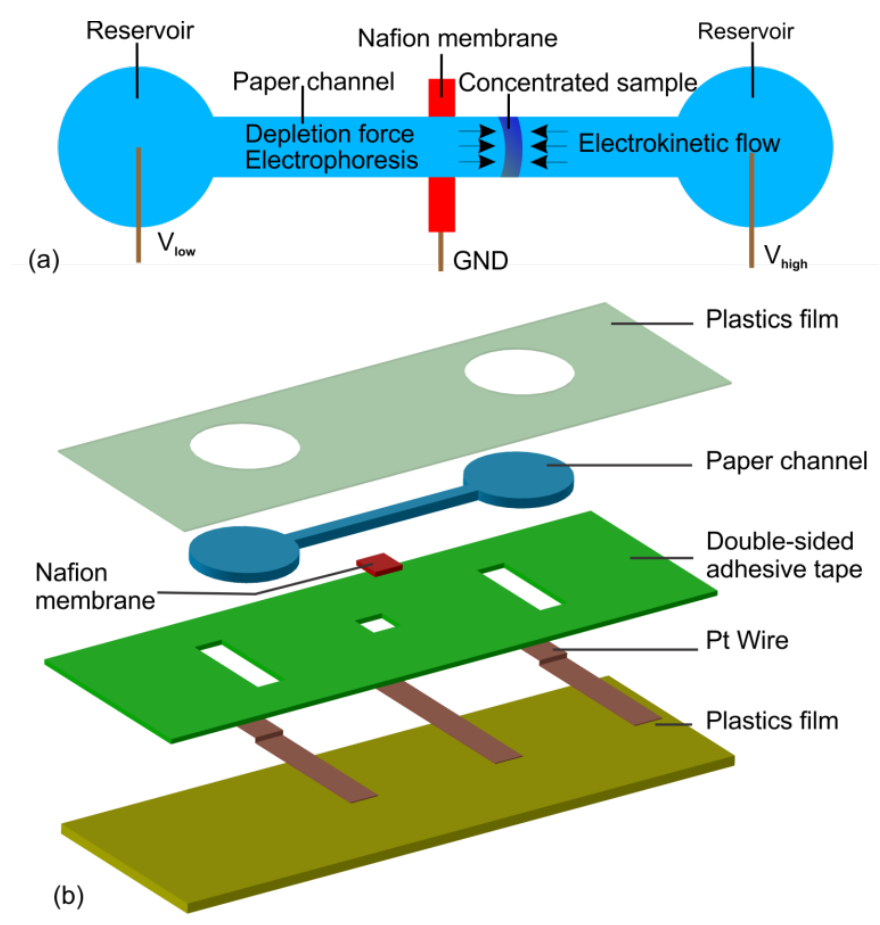

Fig. 1 Paper-based microfluidic device for sample concentration using ion concentration polarization: (a) Working concept; (b) Device structure containing several layers fabricated with different materials.

Figure 1b shows the implementation of the concept depicted in Figure 1a through lamination of multiple layers of the different materials. Each layer of the device was cut in appropriate sizes and dimensions using an electronic craft cutter (Silhouette America, Inc., Silhouette Cameo). ${ }^{26}$ Cellulose filter paper (Whatman) of an average pore size of $11 \mu \mathrm{m}$ (Grade 1) and an average thickness of $180 \mu \mathrm{m}$ was used as the substrate for the paper layer with two circular reservoirs of 1-cm diameter. The strip connecting the reservoirs has a width of $1.5 \mathrm{~mm}$ and a length of $25 \mathrm{~mm}$. The nanoporous Nafion membrane (NRE-212, DuPont, USA) with an average thickness of $50 \mu \mathrm{m}$ was cut in to a $1.5 \mathrm{~mm} \times 5 \mathrm{~mm}$ piece and placed under the paper strip to create the microporous/nanoporous interface. Platinum electrodes with a diameter of $100 \mu \mathrm{m}$ (Sigma-Aldrich) were connected to the two reservoirs and the Nafion membrane. A double-sided adhesive film (thickness of $50 \mu \mathrm{m}$ ) acts as the insulation layer between the electrodes and the paper strip as well as the Nation membrane. In order to establish the electrical connections to the reservoirs and Nafion membrane, the contacts are created by cutting small openings through the adhesive layer. A single-sided $50-\mu \mathrm{m}$ thick plastics film (Scotch, 3M) was used to laminate these layers together. Both top and bottom layers allow to form an enclosure structure and hence prevent evaporation from the paper 
channel. The laminated device was clamped and baked at $110^{\circ} \mathrm{C}$ for 15 minutes. Figure 2 shows the final fabricated device ready for testing.

\section{Materials and methods}

Sodium fluorescence with negative charges (Acid Yellow 73, Sigma Aldrich) with a concentration of $80 \mathrm{mM}$ was used as the sample solution in our experiments. A sample drop was dispensed by an adjustable pipette (Eppendorf Research Plus) into the circular reservoir. The sample is driven into the paper stripe by capillary wetting and is subsequently transported via electrokinetics. For the first experiment to examine the expansion of depletion zone, a DC power supply (Model PS350, Stanford Research System, Inc) provides equal voltages to the platinum wire electrodes. For the second experiment to concentrate the fluorescent dye sample, two independent power sources were used simultaneously to generate a potential difference between two reservoirs. An inverted fluorescence microscope (Eclipse TE2000-E, Nikon) was used to capture the fluorescence images of the concentration zone. All images were taken by a CMOS camera (D700, Nikon) mounted on the front camera port of the microscope. The capture time and exposure time were controlled by using the software Camera Control Pro 2 (Nikon). The recorded images were processed by a customized MATLAB program.

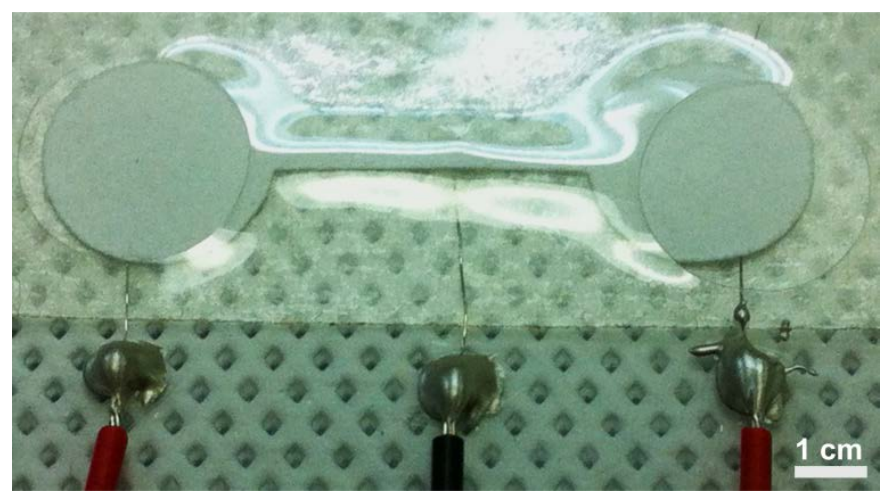

Fig. 2 Fabricated device showing connections with external electrical wires.

\section{Results and discussions}

\section{Expansion of depletion zone}

To start with, a $50 \mu \mathrm{L}$ fluorescence solution was injected into the left reservoir. Then the solution was sucked into the paper strip by capillary force. After two to three minutes, the right reservoir was filled with the solution and the entire channel of the device was pre-wetted (insert in Fig. 3). Once a 50-V voltage was applied to two reservoirs, an ICP depletion zone was seen after a certain time (a few seconds) and it expanded gradually toward the two reservoirs (insert in Fig. 3). The light area in the middle channel region located above the Nafion membrane (connected to GND) shows no presence of fluorescence dye (negative charges) in that depletion zone. As time elapsed, the depletion zone expanded outward such that the fluorescence intensity in the rest of channel became stronger, indicating that fluorescent dye displaced away from the middle depletion region was gradually accumulated in the remaining part of the channel. 
Figure 3 depicts the ICP depletion length over time. The position of the ICP boundary, defined as the distance between the boundary and the edge of the Nafion membrane, was measured every 10 seconds from the recorded images. Such distance was obtained using a customized MATLAB (Mathworks) program. Each fluorescent image was first converted to a binary image where the edge between the enriched and depleted zones was detected. From Figure 3, it shows that the depletion zone expands gradually with time and reaches a steady-state condition after two minutes. The depletion zone initially expands at a rate of $2.7 \mathrm{~mm} / \mathrm{min}$ but gradually slows down to $0.1 \mathrm{~mm} / \mathrm{min}$ two minutes after switching on the voltage. Under a DC electric field, three transport mechanisms are present in such paper channel embedded with the Nafion membrane, including ICP, electrophoresis, and electroosmosis. The ICP causes the ion depletion, thereby pushing the fluorescentt dye to be away from the middle region where the Nafion membrane is placed. Meanwhile, electrophoresis drives the negatively charged fluorescent dye to move towards two reservoirs where positive voltages are applied.

Intriguingly, the effect of electroosmosis is relatively complicated here. In principle, such DC electric field would generate an electroosmotic flow towards the center region of the negatively charged paper channel. Due to the same amount of voltages applied to the two reservoirs, the symmetry will not allow to generate any net flow. So to counterbalance the electroosmosis, a back-pressure is induced, ${ }^{27}$ giving rise to the zero flow rate and hence no bulk flow induced convective transport of the fluorescentt dye. Therefore these three mechanisms lead to the expansion of the depletion zone with increasing time. As for the observed reduction in expansion speed, this phenomenon is resulted from a decrease in the ICP driven fluorescent dye migration with increasing distance from the Nafion membrane and the fluorescent dye acumination induced concentration gradient; both can slow down the depletion zone expansion.

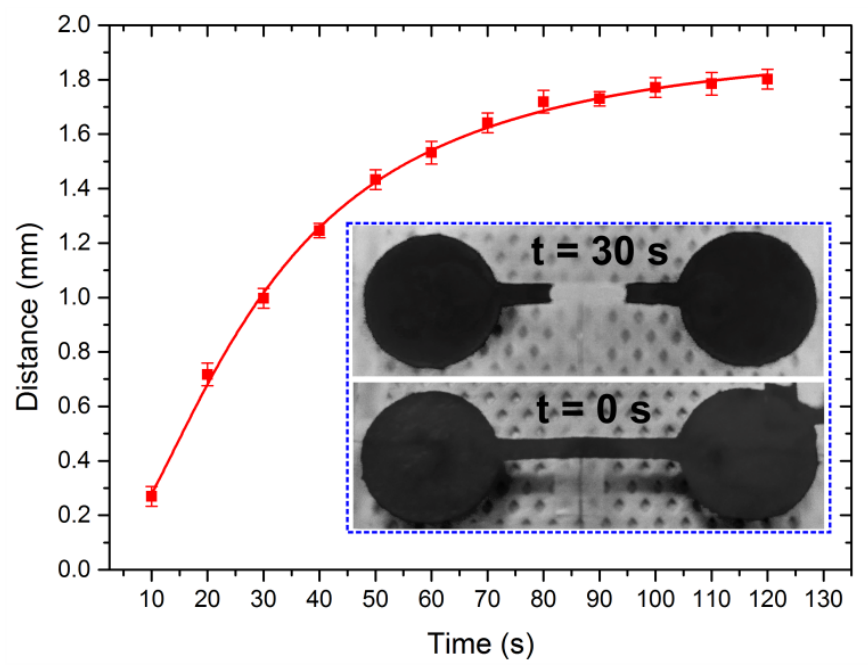

Fig. 3 The expansion of the depletion zone over time. The inserts are converted to black and white images to show the ICP zone. The bottom and top insert correspond to $\mathrm{t}=0$ and $\mathrm{t}=30$ second, respectively.

\section{Sample concentration}

Different from the results shown in Fig. 3 for the expansion of depletion zone generated by imposing the same amount of DC voltages to the two reservoirs, a 
voltage difference is applied across the two reservoirs of the paper strip. The left-hand side reservoir was connected to $50 \mathrm{~V}$, whereas the right-hand side reservoir was connected to $100 \mathrm{~V}$ with the ground electrode underneath the Nafion membrane (see Fig. 1a). Such arrangement of DC voltages caused concentration of the fluorescent dyes, resulting in an enriched band in the paper strip. The band moves toward the reservoir with a higher potential. Such voltage difference would generate a new electroosmotic flow towards to the left-hand side reservoir (with a lower voltage $50 \mathrm{~V}$ applied). It should be noted here that the electroosmotic flow moves in opposite direction to the direction of both ICP and electrophoresis driven motion.

Furthermore, as mentioned earlier, the ICP effect decreases with increasing the distance from the Nafion membrane, the combination of these three transport mechanisms gives rise to a location where the migration velocity of fluorescent dye is zero and hence the enrichment occurs. Such enrichment gets stronger over time, resulting in higher fluorescent intensity of the enriched band with time. Figure 4 depicts the change of the peak fluorescent intensity of the enriched band over time. Within the first 200 seconds, the fluorescent intensity increases almost linearly with time. A peak concentration factor of about 40 times was achieved, comparable to the best performance of the device with the pressed-on nanoporous membrane and better than the in-paper device reported by Gong et al. ${ }^{25}$

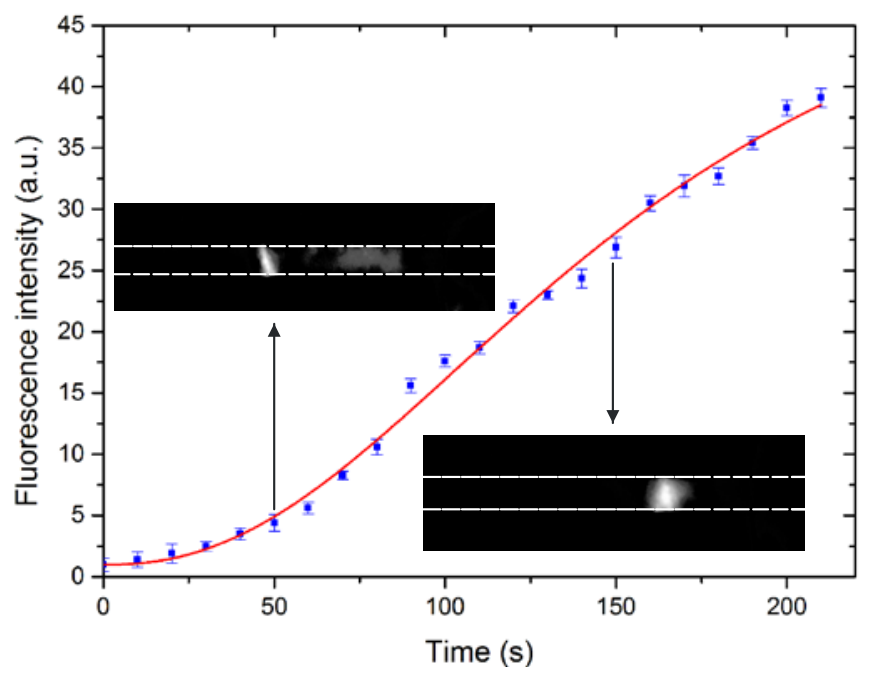

Fig. 4 Maximum fluorescent intensity of the concentrated band versus time.

Furthermore, when the fluorescentt dye was concentrated in an enriched band, it was observed that the band with increasing intensity moves toward the reservoir with the higher potential. This could be due to the finite-reservoir size induced backpressure driven flow that pushes the focused band toward to the opposite direction of the electroosmotic flow (Yan et al. ${ }^{27}$ ). Figure 5(a) shows the representative fluorescent images of the concentrated band at three representative times of 50, 100 and 150 seconds, respectively. The intensity distribution (Fig. 5(b)) shows that not only the intensity (peak) of the band increases but also its area.

To quantify the relative increase of the concentration area over time, we set a threshold value for the intensity of the enriched band to evaluate the band area. Figure 6 shows that the area of the enriched band grows almost exponentially over time. The Taylor dispersion can be the major factor causing this area increment with time. 


\section{Conclusions}

This paper reported a simple fabrication approach for making a paper-based microfluidic platform. Off-the-shelf Nafion membrane was used as a nanoporous junction on the device. The device operated for liquid sample filling by capillary suction through the microporous paper and ion selective transport by DC field driven ICP through the nanoporous Nafion membrane. Two functions of fluorescent dye sample depletion and concentration were successfully demonstrated for the paperbased microfluidic device. When the same potential of $50 \mathrm{~V}$ was imposed to the two reservoirs of the paper strip, the depletion zone expands outward from the Nafion membrane and reaches a steady state after two minutes. When a potential difference was applied with $50 \mathrm{~V}$ to the left-hand side reservoir and $100 \mathrm{~V}$ to the right-hand side reservoir, about 40-folds concentration enhancement was attained within about 200 seconds. The enriched band was observed to slowly move to the reservoir of higher potential with the area of the concentrated band growing over time. The physical mechanisms accounting for the sample depletion and enrichment were discussed, and they mainly include the ICP, electrophoresis and electroosmosis. The lamination approach presented in this paper enables the integration of the concentrator with other paper-based analytical devices. In addition, this technology has the potential to be transferred to a large-scale production using role-to-role technology.

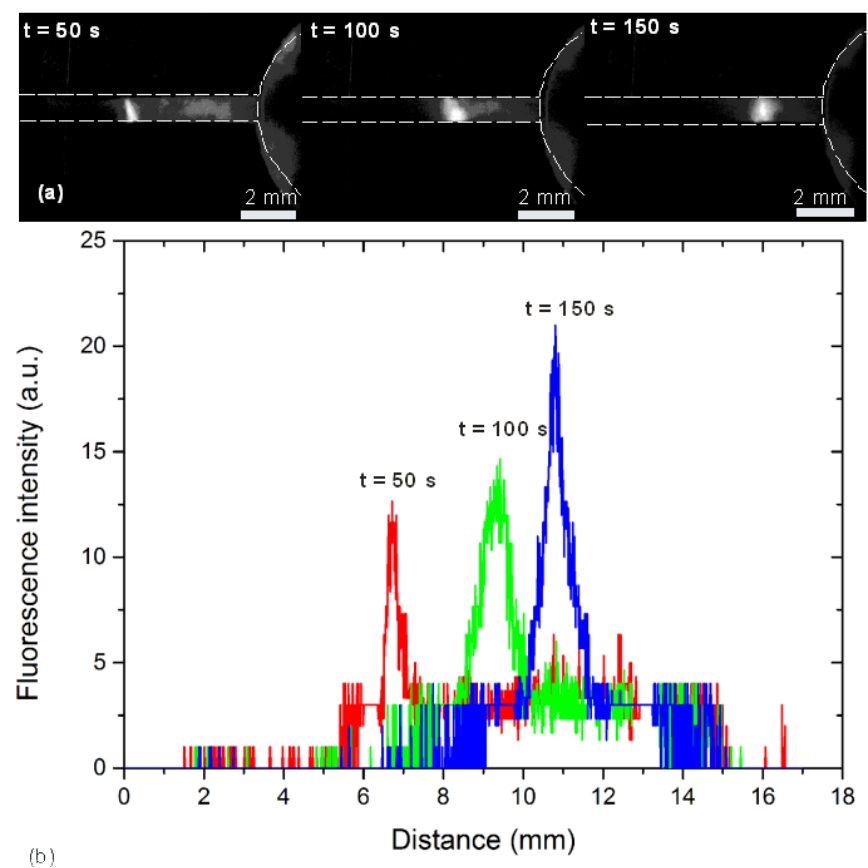

Fig. 5 (a) Grayscale images converted from fluorescent images of the concentrated band at three representative times of $50 \mathrm{~s}, 100 \mathrm{~s}$ and $150 \mathrm{~s}$, respectively. (b) Intensity distributions of the concentrated band at three representative instances of $50 \mathrm{~s}, 100 \mathrm{~s}$ and $150 \mathrm{~s}$. 


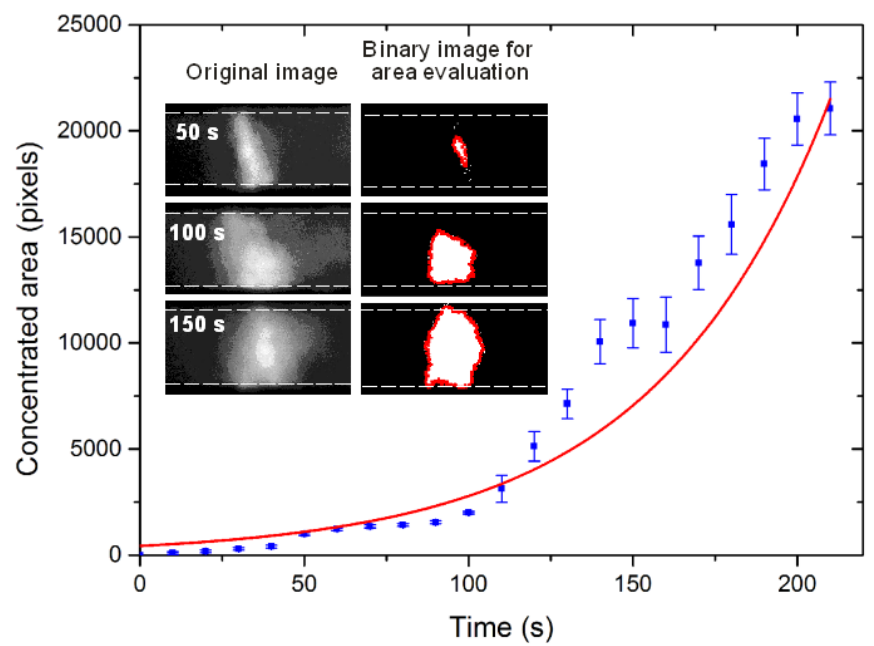

Fig. 6 Area of the concentrated band versus time.

\section{Acknowledgements}

D.T.P acknowledges the support from the Nanyang Technological University (NTU) PhD Scholarship via Nanyang Environment \& Water Research Institute (NEWRI). The authors thank Tuan Tran from School of Mechanical and Aerospace Engineering of NTU for the equipment support.

\section{Notes and references}

${ }^{a}$ School of Mechanical and Aerospace Engineering, Nanyang Technological University, 50 Nanyang Avenue, Singapore 639798. Email: MCYANG@ntu.edu.sg

${ }^{b}$ Harvard-MIT Division of Health Sciences and Technology, Massachusetts Institute of Technology, Cambridge, MA, USA

${ }^{c}$ Queensland Micro- and Nanotechnology Centre, Griffith University, Brisbane, 4111, Australia. E-mail: nam-trung.nguyen@griffith.edu.au

1 Q. Pu, Nano Letters, 2004, 4, 1099-1103.

2 S. J. Kim, Y.-A. A. Song and J. Han, Chemical Society Reviews, 2010, 39, 912-22.

3 S. H. Ko, S. J. Kim, L. F. Cheow, L. D. Li, K. H. Kang and J. Han, Lab on a Chip, 2011, 11, 1351-1358.

4 S. H. Ko, Y.-A. Song, S. J. Kim, M. Kim, J. Han and K. H. Kang, Lab on a Chip, 2012, 12, 4472-82.

5 R. Kwak, S. J. Kim and J. Han, Analytical Chemistry, 2011, 83, 7348-55.

6 S. J. Kim, S. H. Ko, K. H. Kang and J. Han, Nature Nanotechnology, 2010, 5, 297301.

7 B. D. MacDonald, M. M. Gong, P. Zhang and D. Sinton, Lab on a Chip, 2014, 14, 681-685.

8 O. Jannig and N.-T. Nguyen, Microfluidics and Nanofluidics, 2011, 10, 513-519.

9 D. Kim, A. Raj, L. Zhu, R. I. Masel and M. A. Shannon, Lab on a Chip, 2008, 8, 625628.

10 J. Lichtenberg, E. Verpoorte and N. F. de Rooij, Electrophoresis, 2001, 22, 258-271.

11 H. Cui, K. Horiuchi, P. Dutta and C. F. Ivory, 2005, 77, 3451-3457.

12 S. Song, A. K. Singh and B. J. Kirby, Analytical Chemistry, 2004, 76, 4589-4592.

13 R. Oleschuk, L. Shultz-Lockyear, Y. Ning and D. Harrison, Analytical Chemistry, 2000, 72, 585-90.

14 Z. Ge, W. Wang and C. Yang, Lab on a Chip, 2011, 11, 1396-402.

15 S. J. Kim, L. D. Li and J. Han, Langmuir, 2009, 25, 7759-7765. 
16 S. J. Kim, Y.-C.Wang, J. Lee, H. Jang and J. Han, Physical Review Letters, 2007, 99, 044501.

17 A. K. Yetisen, M. S. Akram and C. R. Lowe, Lab on a Chip, 2013, 13, 2210-51.

18 X. Li, D. R. Ballerini and W. Shen, Biomicrofluidics, 2012, 6, 12-14.

19 W. Dungchai, O. Chailapakul and C. S. Henry, Analytical Chemistry, 2009, 81, 58216.

20 A. W. Martinez, S. T. Phillips, G. M. Whitesides and E. Carrilho, Analytical Chemistry, 2010, 82, 3-10.

21 N. Chandra, S. Ali, M. Shaegh, S. Huan, L. Ge and S. Ngin, Sensors and Actuators: B. Chemical, 2014, 204, 414-420.

22 A. K. Ellerbee, S. T. Phillips, A. C. Siegel, K. a. Mirica, A. W. Martinez, P. Striehl, N. Jain, M. Prentiss and G. M. Whitesides, Analytical Chemistry, 2009, 81, 84478452.

23 J. L. Delaney, C. F. Hogan, J. Tian and W. Shen, Analytical Chemistry, 2011, 83, 1300-1306.

24 B. J. Toley, J. A. Wang, M. Gupta, J. R. Buser, L. K. Lafleur, B. R. Lutz, E. Fu and P. Yager, Lab on a Chip, 2015.

25 M. M. Gong, P. Zhang, B. D. MacDonald and D. Sinton, Analytical Chemistry, 2014, 86, 8090-7.

26 E. M. Fenton, M. R. Mascarenas, G. P. Lopez and S. S. Sibbett, ACS Applied Materials and Interfaces, 2009, 1, 124-9.

27 D. G. Yan, C. Yang and X. Y. Huang, Microfluidics and Nanofluidics, 2007, 3, 333-340. 